\title{
Manufactures Human Over-the-Counter Drug Products Not Produced Under an Approved Application or Under a Monograph
}

National Cancer Institute

\section{Source}

National Cancer Institute. Manufactures Human Over-the-Counter Drug Products Not Produced Under an Approved Application or Under a Monograph. NCI Thesaurus. Code C131710.

The process of manufacturing non-prescription medications under neither a monograph nor an approved drug application, for humans by a pharmaceutical company. 